\title{
Le processus de révision en production écrite de textes par des étudiants francophones dyslexiques
}

\author{
Audrey Mazur-Palandre ${ }^{1} 2$ et Florence Chenu ${ }^{3}$ \\ ${ }^{1}$ Université de Lyon \& LabEx ASLAN (ANR-10-LABX-0081, de l'Université de Lyon dans le cadre \\ du programme « Investissements d'Avenir » (ANR-11-IDEX-0007) de l'État Français géré par \\ l'Agence Nationale de la Recherche) \\ ${ }^{2}$ Laboratoire CNRS ICAR (UMR5191, CNRS, Université Lyon 2 et ENS de Lyon) \\ ${ }^{3}$ Laboratoire CNRS DDL (UMR5596, CNRS, Université Lyon 2)
}

\begin{abstract}
Résumé. Une des difficultés mentionnées par les étudiants dyslexiques est la production de textes (Mazur-Palandre, Abadie et Bedoin, 2016). Chez les sujets neurotypiques, les processus orthographiques s'automatisent progressivement avec l'expérience (Berninger et Swanson, 1994), ce qui permettrait - si on se place dans le cadre de la théorie de la capacité pour la production écrite (McCutchen, 1996) - d'affecter davantage de ressources à des processus de niveau supérieur, tels que la révision. Chez les individus dyslexiques, les processus orthographiques sont peu automatisés, et ce même à l'âge adulte ce qui peut donc impacter la production textuelle (Fayol et Miret, 2005). Cette étude vise à analyser les productions écrites d'étudiants dyslexiques et contrôles afin de voir de quelle manière la révision diffère entre les deux groupes d'étudiants. Les étudiants ont été invités à produire des textes écrits, collectés par le biais d'une tablette graphique et du logiciel Eye and Pen $\mathbb{C}$, permettant d'observer la temporalité de l'activité d'écriture, dont les révisions. Les résultats indiquent que les étudiants dyslexiques et contrôles ne diffèrent guère quant à la quantité et les types de révision, ce qui suggère que les étudiants dyslexiques ont mis en place des stratégies. Néanmoins, des analyses complémentaires nuancent les résultats en montrant des différences entre les deux groupes.
\end{abstract}

\begin{abstract}
The revision process during written text production in French dyslexic students? One of the difficulties mentioned by dyslexic students is text production (Mazur-Palandre, Abadie et Bedoin, 2016). In subjects with normal written language development low level processes, such as spelling, become growingly automatized (Berninger \& Swanson, 1994) allowing for allocation of more cognitive resources to higher level processes such as those required for revision. In dyslexic individuals spelling remains poorly automatized even in adults and this may impact compositional performance (Fayol \& Miret, 2005). Thus, this study aims at analyzing the written productions of dyslexic and controls students in order to observe how revision differs between to the two groups. The students were asked to produce written texts collected using a digitizing tablet and
\end{abstract}

\footnotetext{
${ }^{1}$ Corresponding author : audrey.mazur_palandre@ens-lyon.fr
} 
the Eye and Pen $\odot$ software which allows for the observation of the temporality of the writing activity, including revision. The results indicate that dyslexic and control students do not differ much in the amount and types of revisions, suggesting that dyslexic students have developed strategies. Nevertheless, the additional analyzes nuance the quantitative results by showing differences between the two groups.

\section{La dyslexie à l'âge adulte}

La taxonomie internationale de référence, le DSM-5 (American Psychiatric Association, 2013), classe la dyslexie dans l'une des sept catégories des troubles du neurodéveloppement, à savoir dans les troubles spécifiques des apprentissages. Cette catégorie regroupe l'ensemble des signes relevant de troubles de la lecture, de l'écriture et du calcul, sous des critères diagnostiques définis, très globalement, comme suit. Les troubles spécifiques des apprentissages impliqueraient des compétences significativement inférieures à celles attendues pour un âge chronologique donné, interférant négativement et significativement sur les performances académiques. Ces entraves développementales apparaissant durant les années de scolarisation peuvent se manifester seulement lorsque les demandes excèdent les capacités limitées de l'individu, et ne peuvent être expliquées par d'autres déficiences (intellectuelle, auditive, visuelle, etc.) (Habib, 2015, 2018). La manifestation la plus fréquente des troubles spécifiques des apprentissages est la dyslexie, terme qui réfère «à un profil d'apprentissage, caractérisé par des difficultés à reconnaître des mots courants de façon exacte ou fluide et de faibles habilités de décodage et d'orthographe » (Habib, 2018:2, traduit du DSM-5). Les travaux actuels montrent que le périmètre de la définition de la dyslexie peut évoluer (Mazur-Palandre et Witko, 2019a, 2019b). C'est ainsi, par exemple, que la dysorthographie apparait comme un trouble consubstantiel à la dyslexie (Habib, 2003 ; Lyon, Shaywitz et Shaywitz, 2003 ; Snowling, 2000) mais la dyslexie n'est pas seulement un problème d'orthographe (Carter et Sellman, 2013 ; Morken et Helland, 2013). Une des difficultés repérées et mentionnées par les étudiants dyslexiques, concerne la production textuelle. Or les études sur la production verbale orale et écrite des étudiants dyslexiques en termes de syntaxe, de macro-syntaxe et de grammaire de texte sont encore trop rares (Farmer et al., 2002; Morken et Helland, 2013) bien qu'il s'agisse d'une activité très sollicitée à l'Université et particulièrement délicate pour les étudiants atteints de dyslexie/dysorthographie. Les difficultés liées aux processus d'écriture de haut niveau seraient parmi les plus difficiles à saisir (Carter et Sellman, 2013) mais les témoignages des étudiants alertent et montrent qu'elles sont un sujet de préoccupations importantes (Carter et Sellman, 2013 ; Farmer, Riddick et Sterling, 2002 ; Gimenez, Luque, Lopez-Zamora, Fernandes-Navas, 2015 ; Hatcher et al., 2002).

La présente étude a comme objectif d'analyser les productions écrites d'étudiants dyslexiques et d'étudiants contrôles francophones natifs en se focalisant sur un aspect de la production textuelle de haut niveau : les processus de révision. Des études antérieures sur ces mêmes étudiants dyslexiques ont révélé que le système de conversion phonème/graphème n'est pas totalement automatisé (Mazur-Palandre, Abadie et Bedoin, 2016 ; Mazur-Palandre, 2018 , 2019). L'automatisation de ce système de conversion a lieu pour les adultes normoscripteurs durant l'enfance et l'adolescence (Berninger et Swanson, 1994), est ainsi un processus de bas niveau, et requiert moins de ressources cognitives, qui - selon la théorie capacitaire en production écrite (Mc Cutchen, 1996) - se trouvent libérées pour des processus de plus haut niveau tels que la révision de textes (Bereiter et Scardamalia, 1987). Un manque d'automatisation de l'orthographe a un impact négatif sur la composition écrite (Fayol et Miret, 2005). Nous pouvons alors nous demander si ce manque d'automatisation faisant en partie défaut chez les étudiants dyslexiques, a un impact sur un processus de haut niveau 
comme la révision textuelle. Cet article se focalise ainsi sur la révision textuelle afin de déterminer si et en quoi elle est différente selon que les individus sont dyslexiques ou pas.

\section{Production de texte}

\subsection{La complexité de la production écrite dans le cadre de la théorie capacitaire}

L'activité d'écriture est une des tâches cognitives les plus couteuses et complexes (entre autres : Hayes, 1995, 1998 ; Hayes et Flower, 1980 ; Kellogg, 1987, 1994 ; Torrance et Galbraith, 2006) impliquant divers processus cognitifs tels que l'interprétation de texte, la réflexion, la génération d'idées et de structures linguistiques, la résolution des problèmes (planification incluse), la prise de décision ou encore la production d'inférences (Hayes, 1998). Les principaux processus cognitifs identifiés dans la tâche de composition sont la planification, la mise en texte et la vérification (Hayes et Flower, 1980). La planification permet la génération des idées tout en les mettant en relation avec le contexte spécifique de la production textuelle. La mise en texte permet l'élaboration des représentations internes en structures linguistiques et graphiques. Et enfin, la vérification relève d'opérations de contrôle sur le texte. D'autres processus entrent en jeu tels que la résolution de problèmes (planification incluse), la prise de décision et la production d'inférences (Hayes, 1998), l'ensemble des processus se réalisant avec le concours de la mémoire à long terme et de l'environnement de la tâche. Ce modèle, qui avait pour objectif de rendre compte de la production écrite chez l'adulte expert a fait l'objet de nombreuses élaborations, qui ont donné lieu à une version qui permet de tenir compte de la construction progressive de l'expertise rédactionnelle et peut être mise en lien avec des dysfonctionnements (Hayes \& Berninger 2014). En effet, le système de traitement d'information de l'écrit est décrit comme un système à capacité limitée (McCutchen, 1996) : si les ressources cognitives allouées à la transcription restent importantes, les ressources seront moindres pour les autres processus requis tels que la planification ou la mise en texte (Fayol et Miret, 2005 ; McCutchen, 1996).

\subsection{Dyslexie et révision de textes}

Plusieurs études révèlent que les étudiants dyslexiques présentent des difficultés en production écrite, qui peuvent se manifester au niveau du nombre de mots polysyllabiques (Farmer et al., 2002), du nombre d'erreurs d'orthographe (Farmer et al., 2002 ; Hatcher et al., 2002 ; Mazur-Palandre, 2018, 2019 ; Singleton, 1999), de certains aspects syntaxiques (Farmer et al., 2002 ; Mazur-Palandre, 2018, 2019), ou encore de l'identification des erreurs et leurs corrections (Farmer et al., 2002 ; Hatcher et al., 2002 ; Horowitz et Breznitz, 2011). Les étudiants dyslexiques auraient également des difficultés liées au lexique telles que la confusion entre des mots monosyllabiques se ressemblant (which / with; Singleton, 1999), l'omission de mots dans leurs phrases (Singleton, 1999) ou encore l'utilisation d'un vocabulaire inattendu ou inadapté (Farmer et al., 2001 ; Rasking et Higgins, 1995 ; Sterling, Farmer, Riddick, Morgan et Matthews, 1998 ; Singleton, 1999). Ils produiraient également moins rapidement que les étudiants contrôles (Hatcher et al., 2002 ; Singleton, 1999).

Le processus de révision d'un texte écrit est une activité complexe nécessitant une lecture habile de son propre texte. En effet, le scripteur, pour repérer et identifier ses propres erreurs et se corriger, doit traiter visuellement les mots, les analyser phonologiquement et réaliser une association sémantique (Seidenberg et McClelland, 1989). Ces processus peuvent être altérés par un déficit phonologique que les étudiants dyslexiques continuent de présenter (Beaton, McDougall et Singleton, 1997 ; Horowitz-Kraus et Breznitz, 2011 ; Lyon et al., 
1999), par des problèmes d'attention visuelle (Stein, 2001) et des difficultés de traitement syntaxique et sémantique (Rüsseler, Becker, Johannes et Münte, 2007), ce qui pourrait avoir une incidence sur les processus de révision en rendant la relecture d'un texte écrit plus laborieuse (Morken et Helland, 2013). De plus, ces difficultés persistantes à l'âge adulte ont comme effet secondaire un rapport souvent conflictuel et anxiogène à l'écrit, qui peut affecter également l'écrit des personnes dyslexiques (Riddick, Farmer et Sterling, 1997).

Les étudiants dyslexiques francophones natifs présentent encore des difficultés de lecture, que ce soit en lecture de mots isolés ou en lecture de texte (Mazur-Palandre et al., 2016) : ils font plus d'erreurs que les étudiants contrôles et mettent également plus de temps à lire mais font moins d'erreurs si leur temps de lecture n'est pas contraint. Horowitz et Breznitz (2011) suggèrent que les personnes présentant une dyslexie auraient un déficit dans le mécanisme de détection d'erreurs, ne leur permettant alors pas d'identifier toutes les erreurs orthographiques puis de les corriger. En effet, les processus de révision supposent non seulement une détection de l'erreur mais également une correction appropriée : les étudiants dyslexiques auraient non seulement des difficultés à identifier toutes les erreurs mais également à les corriger correctement (Hacker, Plumb, Butterfield, Quathamer et Heiniken, 1994). Farmer et al. (2002) montrent que les étudiants dyslexiques, dans une tâche de révision d'un texte imposé et dans une impliquant la révision de leur propre texte, détectent moins d'erreurs que les étudiants contrôles et qu'ils sont plus susceptibles de repérer les erreurs orthographiques que les erreurs de ponctuation, et plus les erreurs de ponctuation que les erreurs grammaticales. Les étudiants dyslexiques peuvent détecter et corriger certaines de leurs erreurs mais moins efficacement que les étudiants contrôles et dans un temps plus long.

\section{Hypothèses}

Selon la théorie capacitaire (Just et Carpenter, 1992), telle qu'elle est envisagée dans le cas de la production écrite de textes (McCutchen, 1996), les ressources cognitives sont limitées et se répartissent entre les différents processus engagés dans la production textuelle selon leur degré d'automatisation. Un processus largement automatisé de bas niveau requiert ainsi peu de ressources cognitives tandis qu'un processus moins automatisé en appelle davantage. Ainsi, une faible automatisation du système orthographique exige une forte mobilisation des ressources au détriment de processus de haut niveau tel que les processus de révision (McCutchen, 1996). Afin de contribuer aux travaux sur les processus de révision des étudiants dyslexiques, nous avons donc analysé les révisions auxquelles ils procèdent dans leurs propres textes. Nous faisons l'hypothèse que les étudiants dyslexiques révisent moins leur texte que les étudiants contrôles (H1, ce qui confirmerait les travaux de Farmer et al., 2002) mais présentent un pattern de type de révisions identique aux étudiants contrôles (H2, ce qui confirmerait les travaux de Farmer et al., 2002). Néanmoins, des analyses complémentaires plus fines, nous attendons que les étudiants dyslexiques présentent des erreurs de détection et de correction des erreurs (H3). De plus, une analyse d'un questionnaire centré sur leurs difficultés et ressentis est également présentée, afin d'approcher l'insécurité linguistique que ces étudiants peuvent manifester.

\section{Méthode}

\subsection{Participants}


La collecte des données a été réalisée dans le cadre de projets PEPS CNRS ${ }^{2}$ concernant les difficultés et besoins des étudiants dyslexiques dans l'enseignement supérieur. Dans un premier temps, deux questionnaires en ligne ont été remplis par 1450 étudiants pour le premier et 1472 pour le second. Dans ce présent article, seront présentés les résultats du second questionnaire, qui portait en partie sur le ressenti des difficultés en lien avec la production écrite. Afin de pouvoir faire la différence entre ce qui correspond à une difficulté commune à la majorité des étudiants et ce qui est plus spécifique aux étudiants dyslexiques, 83 étudiants dyslexiques et 83 étudiants contrôles appariés en genre, âge et niveau d'étude ont été sélectionnés parmi les 1472 (Tableau 1). Les étudiants dyslexiques présentant un trouble de l'attention avec ou sans hyperactivité (TDAH) ou d'autres types de pathologies ont été exclus de la présente étude.

Tableau 1. Description des groupes d'individus ayant répondu au questionnaire.

\begin{tabular}{lcc}
\hline & Étudiants dyslexiques & Étudiants contrôles \\
\hline Âge & 39 & \\
\hline Moins de 20 ans & 27 & 39 \\
\hline $20-23$ ans & 17 & 27 \\
24 ans et plus & $\mathbf{8 3}$ & 17 \\
\hline Nombre total d'individus & 50 femmes $/ 33$ hommes & 50 femmes $/ 33$ hommes \\
\hline Selon le genre & & \\
\hline
\end{tabular}

Parmi tous les étudiants ayant participé aux deux questionnaires, une trentaine d'étudiants dyslexiques et une trentaine d'étudiants contrôles appariés en âge, genre et niveau d'études ont accepté de passer un bilan orthophonique et neuropsychologique puis une tâche psycholinguistique de production de textes. Ces données textuelles, qui sont traitées dans ce présent article, de 21 étudiants dyslexiques et 22 contrôles ont été analysées (Tableau 2).

Tableau 2. Description des groupes d'individus ayant participé à la tâche de production de textes.

\begin{tabular}{lcc}
\hline & Étudiants dyslexiques & Étudiants contrôles \\
\hline Âge moyen & 21,7 & 21,8 \\
\hline Intervalle & {$[18 ; 1-28 ; 5]$} & {$[18 ; 1-28 ; 9]$} \\
\hline Nombre total d'individus & $\mathbf{2 1}$ & $\mathbf{2 2}$ \\
\hline Selon le genre & 9 femmes $/ 12$ hommes & 10 femmes $/ 12$ hommes \\
\hline
\end{tabular}

Les individus dyslexiques retenus, diagnostiqués durant l'enfance, ont tous une dysorthographie associée et ont suivi au cours de leur enfance/adolescence des rééducations. Lors de la collecte des données, seuls deux étudiants dyslexiques parmi les 21 participants, disaient être déclarés à la Mission Handicap de leur établissement et ainsi bénéficier d'un tiers temps de composition supplémentaire lors des épreuves écrites (aucun ne disposait donc d'outils numériques spécifiques ou ne suivait un programme de remédiation au moment de la collecte). Les étudiants sont tous monolingues et francophones natifs et ont signé un formulaire de consentement pour participer au bilan et à la tâche psycholinguistique de production de textes. Les critères d'exclusion, vérifiés à l'anamnèse (lors du bilan), écartaient les individus présentant des déficits auditifs ou visuels, ou d'autres troubles.

\subsection{Collecte des données}

\subsubsection{Les enquêtes}

\footnotetext{
2 Projets ETUDYS et DYS'R'ABLE, co-financés par le LabEx ASLAN, les laboratoires DDL (UMR 5596 CNRS et Université Lyon 2) et ICAR (CNRS, Université Lyon 2 et ENS de Lyon).
} 
Un premier objectif était de connaître les besoins et difficultés des étudiants dyslexiques de l'Université de Lyon. C'est dans cette mesure que des enquêtes sous forme de questionnaire en ligne ont été élaborées et diffusées. Les enquêtes ont été élaborées par un groupe pluridisciplinaire (chercheurs, enseignants-chercheurs, chargés de mission handicap, associations, orthophonistes, neuropsychologues). Le premier questionnaire ETUDYS abordait plusieurs thèmes, du diagnostic à la vie universitaire en passant par les apprentissages. Le second, DYS'R'ABLE, se focalisait sur la production textuelle à l'orale et à l'écrit et sur l'apprentissage de l'anglais.

\subsubsection{Le bilan orthophonique et neuropsychologique}

Parmi les étudiants ayant répondu aux enquêtes (dyslexiques ou non), une trentaine a ensuite été contactée afin de participer à un bilan orthophonique et neuropsychologique, qui a été pris en charge par une psychologue-neuropsychologue et une enseignante-chercheure en psychologie . Le bilan a débuté par une anamnèse en entretien individuel. Le traitement du langage écrit était évalué avec des épreuves de la batterie ECLA 16+ (Gola-Asmussen, Lequette, Pouget, Rouyer et Zorman, 2010) et du Vol du PC (Boutard, Claire et Gretchanovsky, 2004). Le décodage a ainsi été testé avec la lecture de mots isolés (mots réguliers, irréguliers et pseudo-mots), la lecture à haute voix de textes avec (Le vol du PC) et sans véritable signification (L'Alouette). L'orthographe était évaluée en dictée de mots isolés (mots réguliers, irréguliers et pseudo-mots) et de texte (ECLA 16+). La compréhension en lecture était mesurée à l'aide de subtests du Vol du PC : épreuves récit (résumer le texte), questions ouvertes, QCM, choix des titres et recherche d'informations. Les compétences méta-phonologiques étaient également évaluées. Des tests neuropsychologiques issus de la TAP-M (Zimmermann et Fimm, 2012) estimaient plusieurs aspects de l'attention et des fonctions exécutives. Enfin, des compétences visuo-attentionnelles étaient évaluées avec le test du Report Global pour l'empan visuo-attentionnel (Bosse, Tainturier et Valdois, 2007 ; Bosse et Valdois, 2009, voir EVADYS (Valdois, Guinet, et Embs, 2014)), le test SIGL pour les traitements global/local (Bedoin et Médina, 2015), un test de recherche visuelle (Barrage de n, ECLA 16+) et des épreuves d'orientation visuelle et auditive (Visioner et Audioner (Bedoin, 2014)). Deux épreuves des échelles de Wechsler évaluaient le raisonnement perceptif (Matrices), la mémoire à court terme et la mémoire de travail auditivo-verbales (Mémoire des chiffres)s.

\subsubsection{Tâche psycholinguistique : production de textes}

Protocole. À la suite des bilans orthophonique et neuropsychologique, les étudiants ont effectué la tâche psycholinguistique, durant laquelle il a été demandé aux étudiants de produire quatre textes sur la thématique du conflit entre les gens ${ }^{6}$, qui correspondent à quatre conditions expérimentales : narratif oral, narratif écrit, expositif oral et expositif écrit. La collecte s'est organisée en deux sessions séparées d'une semaine. Lors de la Session 1, les individus visionnaient une vidéo illustrative de la thématique puis produisaient deux textes (narration ou exposition, à l'écrit et à l'oral) séparés par une tâche de distraction. Lors de la Session 2, les participants produisaient les deux autres textes. Les individus ont donc été répartis de manière équilibrée en deux ordres : la moitié produisait un texte écrit puis un oral et l'autre moitié un texte oral puis un écrit. Le type de texte a également un effet significatif

\footnotetext{
${ }^{3}$ Raphaëlle Abadie, Psychologue-Neuropsychologue à l'Hôpital Femme Mère Enfant de Bron (69) et au Centre d'Action Médico-Sociale Précoce de Décines (69).

${ }^{4}$ Nathalie Bedoin, MCF, Université Lyon 2 et Laboratoire DDL (UMR 5596)

${ }^{5}$ Pour plus d'information sur les résultats concernant le bilan orthophonique et neuropsychologique, cf. Mazur-

Palandre, Abadie et Bedoin (2016) et Abadie et Bedoin (2016).

${ }^{6}$ Vidéo créée pour le projet Spencer (fondation Spencer, USA), porteur Ruth Berman.
} 
pour de nombreux critères d'analyses (entre autres, Berman, 1998 ; Berman et Nir-Sagiv, 2009 ; Chenu et al, 2012 ; Jisa, 2004, 2016 ; Mazur-Palandre et Jisa, 2013), dans la mesure où les textes narratif et expositif s'opposent par leurs structures, leurs contenus et leurs buts communicationnels (Mosenthal, 1985).

Consignes. Pour le texte expositif, il était demandé aux étudiants de produire un texte sur les problèmes entre les gens, de discuter de cette thématique en présentant leurs idées comme lors d'un exposé scolaire. Pour le texte narratif, il était demandé aux étudiants de raconter une histoire personnelle retraçant un conflit qu'ils auraient pu vivre.

\subsection{Traitement des données psycholinguistiques}

\subsubsection{Traitements des données}

Les données orales ont été collectées par le biais d'un enregistreur audio et d'une caméra vidéo. Elles ont ensuite été numérisées puis transcrites sous le logiciel Transcriber. Les données écrites ont été collectées par le biais de tablettes graphiques via le logiciel Eye and Pen $\odot$ (Chesnet et Alamargot, 2005). Les transcriptions des données orales et écrites ont été réalisées en respectant les conventions CHILDES (http://childes.psy.cmu.edu/) afin de pouvoir les exporter, grâce à des filtres adaptés ${ }^{7}$, dans le logiciel CLAN, dans lequel ces productions orales et écrites ont pu être codées et traitées de façons similaires. Les productions ont été découpées en clauses (ou propositions) - la clause se définissant comme une unité de signification constituée d'un verbe fini ou non fini et d'arguments - et en unités terminales (UT) - unité étant composée d'une clause principale et de toutes ses clauses dépendantes telles que ses subordonnées ; ces deux unités apparaissant comme de bons critères pour l'évaluation du développement syntaxique (Berman, 1998 ; Hunt, 1970).

Notre corpus est constitué de 172 textes, 86 oraux et 86 écrits. Pour cet article, nous retenons les 86 textes écrits, ce qui représente 2328 clauses (expositifs : 1089 ; narratifs : 1239) et 1126 UT (expositifs : 515 ; narratifs : 611). Le Tableau (3) donne des indications de longueur des textes selon le type de texte produit et le groupe.

Tableau 3. Indicateurs de longueur des textes écrits selon le type de texte et le groupe

\begin{tabular}{lllll}
\hline & Textes expositifs & \multicolumn{3}{l}{ Textes narratifs } \\
\cline { 2 - 5 } & Étu. Dyslexiques & Étu. Contrôles & Étu. Dyslexiques & Étu. Contrôles \\
\hline Nombre de mots par texte & $198,2(101)$ & $181,1(139)$ & $207(131)$ & $181(112)$ \\
\hline Nombre de clauses par texte & $25,8(12,7)$ & $25(17)$ & $30,7(20)$ & $27(16)$ \\
\hline Nombre de UT par texte & $12(5)$ & $12(9,4)$ & $14,7(9,9)$ & $13,8(8,2)$ \\
\hline Nombre de clauses par UT & $2,1(0,5)$ & $2,2(0,4)$ & $2,1(0,3)$ & $2,04(0,5)$ \\
\hline
\end{tabular}

Les analyses ANOVA montrent que les différences de longueur entre les étudiants dyslexiques et contrôles en nombre de mots $\left(\mathrm{F}_{(1,39)}=0,089, \mathrm{p}=.767\right)$, de clauses $\left(\mathrm{F}_{(139)}=1,842, \mathrm{p}=\right.$ $.183)$, d'Unités $\mathrm{T}\left(\mathrm{F}_{(139)}=2,501, \mathrm{p}=.122\right)$ et de clauses par Unité $\mathrm{T}\left(\mathrm{F}_{(139)}=0,773, \mathrm{p}=.385\right)$ ne sont pas significatives.

\subsubsection{Codage des révisions}

Une fois les transcriptions réalisées entièrement, toutes les révisions réalisées par les étudiants ont été codées dans le logiciel Eye and Pen®), puis les données ont été transférées dans CLAN, afin que nous puissions profiter des commandes de comptage. Ont été considérés comme révision tous les mouvements impliquant un retour en arrière (excepté pour les accents ou les points sur les $« \mathrm{i} », \ll \mathrm{j} »$ ou les barres $\mathrm{du} \ll \mathrm{t} »)$. 
Chaque révision est codée selon trois paramètres : (1) l'endroit dans le texte à partir duquel la révision a lieu ; (2) l'unité sur laquelle la révision a lieu et (3) enfin le type et le contenu de la révision. Ces trois paramètres sont reliés par un indice : le numéro d'ordre de la révision.

En ce qui concerne le type et le contenu de la révision, trois informations sont codées : (1) la distance entre l'endroit dans le texte à partir duquel la révision a lieu et l'unité affectée par la révision (cette distance est codée comme longue si la révision intervient après que le texte ait été entièrement rédigé, moyenne si la révision a lieu sur une unité qui remonte audelà d'une frontière syntaxique ou courte si elle intervient sur le mot en train d'être écrit ; (2) la nature de la révision, qui peut être une suppression (les problèmes entre les gens), une reformulation (les problèmes entre les gens personnes), sans changement (tesles problèmes), une insertion (les + principaux problèmes) ou un déplacement (les problèmes principaux -> les principaux problèmes) ; et (3) la portée de la révision, qui peut être indéterminée, esthétique, de l'ordre de la ponctuation, du soulignement, de l'orthographe lexicale, de l'orthographe grammaticale, du mot, du constituant, de la clause, de la phrase ou de l'épisode.

(1) Exemple d'une révision de longueur moyenne, dont la nature est une insertion, et la portée de l'ordre de l'orthographe grammaticale (étudiant dyslexique, $n^{\circ} 43$ )

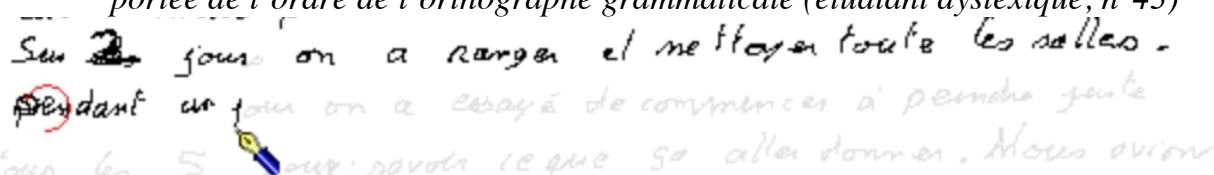

L'étudiant a écrit : «Sur les 2 jour on a ranger et nettoyer toute les salles pendant un jour », une fois le mot « jour » écrit (deuxième occurrence), il s'arrête puis retourne sur la ligne d'audessus pour insérer un « $\mathrm{s} »$ de pluriel à la première occurrence du mot « jour ».

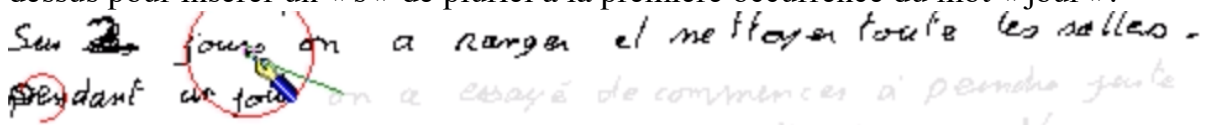

Tous les textes ont été codés par un premier codeur, puis ils ont été recodés par un second codeur. Pour les cas problématiques, les décisions étaient prises en équipe.

\section{Résultats}

Certains résultats du questionnaire du projet DYS'R'ABLE sont présentés (analyses statistiques CHI 2). Concernant les révisions des textes écrits, des analyses de variance (comparaison de moyennes, ANOVA) ont été effectuées avec : (a) deux facteurs interindividuels, le groupe (étudiants dyslexiques et contrôles) ; et (b) un facteur intraindividuel, le type de texte (expositif et narratif). Les analyses ont été réalisées sur: la proportion de révisions, la proportion de révisions selon leur type (suppression, remplacement, insertion - les autres types ayant une trop faible proportion pour des analyses), leur distance (courte, moyenne et longue) et leur nature (proportion des révisions selon leur type, esthétique, de l'ordre de la ponctuation, de l'orthographe, du mot ou supérieur au mot). Seules les interactions significatives sont présentées.

\subsection{Analyses du questionnaire}

Lors du questionnaire DYS'R'ABLE, les étudiants avaient à répondre à plusieurs questions concernant leur diagnostic, la production écrite et orale et enfin l'apprentissage de l'anglais. Nous nous intéresserons aux questions concernant la production orale et écrite. Les analyses ont été réalisées sur 83 étudiants contrôles et 83 étudiants dyslexiques, appariés en âge, genre et niveau d'études. Le tableau 4 présente les résultats significatifs concernant les questions sur la production écrite : les tests, pour chacune des difficultés mentionnées, fournissent un p inférieur à 0,01 révélant que l'appréciation de cette difficulté est liée au type de population. 
Tableau 4. Résultats significatifs des analyses du questionnaire selon le groupe

\begin{tabular}{|c|c|}
\hline Difficultés & Test CHI 2 \\
\hline $\begin{array}{l}78,2 \% \text { des étudiants dyslexiques déclarent avoir des difficultés à organiser } \\
\text { leur discours, contre seulement } 21,5 \% \text { des étudiants contrôles. }\end{array}$ & $\chi^{2}=50,4, p<0,001$ \\
\hline $\begin{array}{l}16,5 \% \text { des étudiants dyslexiques déclarent avoir systématiquement des } \\
\text { difficultés à organiser un plan, contre seulement } 2,5 \% \text { des contrôles. }\end{array}$ & $\chi^{2}=11,7, p<0,05$ \\
\hline $\begin{array}{l}59,7 \% \text { des étudiants dyslexiques déclarent avoir systématiquement ou } \\
\text { fréquemment des difficultés avec le temps et le mode des verbes, contre } 18 \% \\
\text { des étudiants contrôles. }\end{array}$ & $\chi^{2}=32,6, \mathrm{p}<0,0001$ \\
\hline $\begin{array}{l}42,7 \% \text { des étudiants dyslexiques déclarent n'avoir jamais de difficultés avec } \\
\text { la ponctuation, contre } 74,4 \% \text { des étudiants contrôles. }\end{array}$ & $\chi^{2}=19,9, \mathrm{p}<0,0001$ \\
\hline $\begin{array}{l}67,6 \% \text { des étudiants dyslexiques déclarent systématiquement ou fréquemment } \\
\text { diviser leur texte en paragraphes, contre } 85,5 \% \text { des contrôles. }\end{array}$ & $\chi^{2}=22,5, \mathrm{p}<0,0001$ \\
\hline $\begin{array}{l}73,7 \% \text { des étudiants dyslexiques déclarent avoir systématiquement ou } \\
\text { fréquemment des difficultés avec l'orthographe, contre } 14,7 \% \text { des contrôles. }\end{array}$ & $\begin{array}{l}\chi^{2}=75,95, \mathrm{p}< \\
0,0001\end{array}$ \\
\hline $\begin{array}{l}75,6 \% \text { des étudiants dyslexiques déclarent avoir systématiquement ou } \\
\text { fréquemment peur de faire des fautes d'orthographe, contre } 33,8 \% \text { des } \\
\text { étudiants contrôles. }\end{array}$ & $\chi^{2}=33,9, \mathrm{p}<0,0001$ \\
\hline $\begin{array}{l}28 \% \text { des étudiants dyslexiques déclarent avoir systématiquement ou } \\
\text { fréquemment l'impression de manquer de vocabulaire et } 48,8 \% \text { de temps } \\
\text { en temps, contre } 22 \% \text { des étudiants contrôles systématiquement ou } \\
\text { fréquemment et } 39 \% \text { de temps en temps. }\end{array}$ & $\chi^{2}=8,4, p<0,05$ \\
\hline $\begin{array}{l}34,6 \% \text { des étudiants dyslexiques déclarent qu'ils n'ont jamais de difficultés à } \\
\text { organiser les mots dans une phrase contre } 65,1 \% \text { des étudiants contrôles. }\end{array}$ & $\chi^{2}=17,2, \mathrm{p}<0,0001$ \\
\hline $\begin{array}{l}43,9 \% \text { des étudiants dyslexiques disent pouvoir se concentrer jusqu'à } 30 \\
\text { minutes sans interruption (contre } 28,6 \% \text { des étudiants contrôles) et seulement } \\
6,1 \% \text { plus de } 2 \text { heures (contre } 29,9 \% \text { pour plus de } 2 \text { heures). }\end{array}$ & $\chi^{2}=16,7, p<0,0001$ \\
\hline $\begin{array}{l}51 \% \text { des étudiants dyslexiques déclarent interrompre systématiquement ou } \\
\text { fréquemment l'activité d'écriture à cause de la fatigue contre } 33 \% \text { des } \\
\text { étudiants contrôles. }\end{array}$ & $\chi^{2}=9,4, p<0,05$ \\
\hline $\begin{array}{l}\text { 63,9\% des étudiants dyslexiques déclarent interrompre systématiquement ou } \\
\text { fréquemment l'activité d'écriture à cause du bruit contre } 29,2 \% \text { des étudiants } \\
\text { contrôles. }\end{array}$ & $\chi^{2}=20,3, \mathrm{p}<0,0001$ \\
\hline
\end{tabular}

Les analyses du questionnaire DYS'R'ABLE mettent en évidence que les étudiants dyslexiques sont significativement plus dans une insécurité à l'écrit que les étudiants contrôles. Ils déclarent se sentir en difficulté que ce soit en macrostructure (organisation du texte, mise en place d'un plan, découpage du texte en paragraphes, etc.) ou en microstructure (temps et mode des verbes, organisation des unités dans les phrases), dans l'accès lexical (sentiment de manquer de vocabulaire) ou en orthographe (peur de faire des erreurs et difficultés à gérer l'orthographe). Ces résultats confirment des travaux antérieurs qui montrent également que les étudiants dyslexiques ne sentent pas à l'aise lors de tâches de production textuelle et ressentent encore de grandes difficultés en orthographe, syntaxe et organisation textuelle (Gimenez et al., 2015 ; Jacquier et al., 2009) et ces difficultés sont bien confirmées par des expérimentations (Gimenez et al., 2015 ; Gregg, Coleman et Lindstrom, 2008 ; Farmer, et al., 2002 ; Hatcher et al., 2002). Ces analyses du questionnaire révèlent également que les étudiants dyslexiques ressentent plus de difficultés que les étudiants contrôles à se concentrer longuement lors d'une tâche textuelle : près de $30 \%$ des étudiants contrôles disent pouvoir se concentrer plus de deux heures, contre seulement $6,1 \%$ des étudiants dyslexiques. La majorité des étudiants dyslexiques disent ne pas pouvoir se concentrer plus de 30 minutes lors de la rédaction d'un texte sans interruption. De plus, le questionnaire montre que les étudiants dyslexiques sont plus sensibles à la fatigue et les bruits que les étudiants contrôles. Ces ressentis sont confirmés par des travaux antérieurs portant sur l'attention des étudiants dyslexiques, qui mettent en évidence que les étudiants 
dyslexiques, s'ils n'ont pas un déficit attentionnel, ont néanmoins un système attentionnel atypique (Bedoin et Abadie, 2016 ; Mazur-Palandre et al., 2016).

\subsection{Analyses quantitatives des révisions}

\subsubsection{Proportion de révisions}

Un test de comparaison de moyennes (ANOVA) a été effectué sur la proportion de révisions (selon le nombre de mots par texte), selon les facteurs groupe et type de texte (Tableau 5).

Tableau 5. Proportion de révisions par texte selon le groupe et le type de texte

\begin{tabular}{lclllll}
\hline Facteurs & $\mathrm{N}=$ & & Moyennes & Écarts-types & ANOVA & \\
\hline GROUPE & 43 & Dys. & 0,063 & 0,03 & $\mathrm{~F}_{(a, A)}=2,053, \mathrm{p}=.159$ & NS \\
& & Contrôle & 0,05 & 0,03 & & \\
\hline TYPE DE & 43 & Expo. & 0,057 & 0,03 & $\mathrm{~F}_{(a, t)}=0,220, \mathrm{p}=.641$ & NS \\
\cline { 3 - 7 } TEXTE & & Narr. & 0,054 & 0,03 & & \\
\hline
\end{tabular}

Les résultats montrent qu'il n'y a pas de différence significative entre les deux groupes et les types de textes.

\subsubsection{Nombre de révisions selon leur type}

Des tests de comparaison de moyennes (ANOVA) ont été effectués sur les proportions de révisions (selon le nombre de mots par texte), selon leur type et selon les facteurs groupe et type de texte (Tableau 6).

Tableau 6. Proportion de révisions par texte selon leur type et les facteurs GROUPE et TYPE DE TEXTE

\begin{tabular}{|c|c|c|c|c|c|c|}
\hline Facteurs & $\mathrm{N}=$ & & Moyennes & Écart-type & ANOVA & \\
\hline \multicolumn{7}{|c|}{ Révision de type « suppression» } \\
\hline \multirow[t]{2}{*}{ GROUPE } & \multirow[t]{2}{*}{43} & Dys. & 0,065 & 0,01 & \multirow[t]{2}{*}{$\mathrm{F}_{(\mathrm{A} A)}=0,390, \mathrm{p}=.536$} & \multirow[t]{2}{*}{ NS } \\
\hline & & Contrôle & 0,007 & 0,01 & & \\
\hline \multirow{2}{*}{$\begin{array}{l}\text { TYPE DE } \\
\text { TEXTE }\end{array}$} & \multirow[t]{2}{*}{43} & Expo. & 0,01 & 0,01 & \multirow[t]{2}{*}{$\mathrm{F}_{(a, A)}=1,298, \mathrm{p}=.261$} & \multirow[t]{2}{*}{ NS } \\
\hline & & Narr. & 0,009 & 0,0008 & & \\
\hline \multicolumn{7}{|c|}{ Révision de type « reformulation» } \\
\hline \multirow[t]{2}{*}{ GROUPE } & \multirow[t]{2}{*}{43} & Dys. & 0,021 & 0,01 & \multirow[t]{2}{*}{$F_{(a, A)}=6,539, p=.014$} & \multirow[t]{2}{*}{$\mathbf{S}$} \\
\hline & & Contrôle & 0,012 & 0,01 & & \\
\hline \multirow{2}{*}{$\begin{array}{l}\text { TYPE DE } \\
\text { TEXTE }\end{array}$} & \multirow[t]{2}{*}{43} & Expo. & 0,015 & 0,01 & \multirow[t]{2}{*}{$\mathrm{F}_{(a, A)}=1,298, \mathrm{p}=.261$} & \multirow[t]{2}{*}{ NS } \\
\hline & & Narr. & 0,018 & 0,0008 & & \\
\hline \multicolumn{7}{|c|}{ Révision de type « insertion» } \\
\hline \multirow[t]{2}{*}{ GROUPE } & \multirow[t]{2}{*}{43} & Dys. & 0,015 & 0,01 & \multirow[t]{2}{*}{$\mathrm{F}_{(a .41)}=0,887, \mathrm{p}=.352$} & \multirow[t]{2}{*}{ NS } \\
\hline & & Contrôle & 0,019 & 0,01 & & \\
\hline \multirow{2}{*}{$\begin{array}{l}\text { TYPE DE } \\
\text { TEXTE }\end{array}$} & \multirow[t]{2}{*}{43} & Expo. & 0,019 & 0,03 & \multirow[t]{2}{*}{$\mathrm{F}_{(a, A)}=1,288, \mathrm{p}=.263$} & \multirow[t]{2}{*}{ NS } \\
\hline & & Narr. & 0,015 & 0,03 & & \\
\hline
\end{tabular}

Les résultats montrent que seul le facteur GROUPE a un effet significatif sur la proportion d'un type de révision, la reformulation, le fait de remplacer une unité par une autre (2).

(2) Mais devant l'incivilité absolue de <mes $>$ ces jeunes [...] » (étudiant contrôle $n^{\circ} 46$, texte narratif)

Les étudiants dyslexiques en réalisent davantage que les étudiants contrôles.

\subsubsection{Nombre de révisions selon la distance}


Des tests de comparaison de moyennes (ANOVA) ont été effectués sur les proportions de révisions (selon le nombre de mots par texte), selon leur type et selon les facteurs groupe et type de texte (Tableau 7).

Tableau 7. Proportion de révisions par texte selon leur distance et les facteurs GROUPE et TYPE DE

\begin{tabular}{|c|c|c|c|c|c|c|}
\hline Facteurs & $\mathrm{N}=$ & & Moyennes & Écart-type & ANOVA & \\
\hline \multicolumn{7}{|c|}{ Révision de distance « courte $»$} \\
\hline \multirow[t]{2}{*}{ GROUPE } & \multirow[t]{2}{*}{43} & Dys. & 0,046 & $0,01-$ & \multirow[t]{2}{*}{$\mathrm{F}_{(a A)}=0,830, \mathrm{p}=.368$} & \multirow[t]{2}{*}{ NS } \\
\hline & & Contrôle & 0,037 & 0,02 & & \\
\hline \multirow{2}{*}{$\begin{array}{l}\text { TYPE DE } \\
\text { TEXTE }\end{array}$} & \multirow[t]{2}{*}{43} & Expo. & 0,042 & 0,02 & \multirow[t]{2}{*}{$\mathrm{F}_{(a, A)}=0,015, \mathrm{p}=.902$} & \multirow[t]{2}{*}{ NS } \\
\hline & & Narr. & 0,043 & 0,02 & & \\
\hline \multicolumn{7}{|c|}{ Révision de distance « moyenne » } \\
\hline \multirow[t]{2}{*}{ GROUPE } & \multirow[t]{2}{*}{43} & Dys. & 0,008 & 0,01 & \multirow{2}{*}{$\mathrm{F}_{(1, A)}=0,701, \mathrm{p}=.407$} & \multirow[t]{2}{*}{ NS } \\
\hline & & Contrôle & 0,006 & 0,01 & & \\
\hline \multirow{2}{*}{$\begin{array}{l}\text { TYPE DE } \\
\text { TEXTE }\end{array}$} & \multirow[t]{2}{*}{43} & Expo. & 0,008 & 0,01 & \multirow[t]{2}{*}{$\mathrm{F}_{(a, A)}=0,863, \mathrm{p}=.358$} & \multirow[t]{2}{*}{ NS } \\
\hline & & Narr. & 0,005 & 0,01 & & \\
\hline \multicolumn{7}{|c|}{ Révision de distance « longue » } \\
\hline \multirow[t]{2}{*}{ GROUPE } & \multirow[t]{2}{*}{43} & Dys. & 0,004 & 0,01 & \multirow[t]{2}{*}{$\mathrm{F}_{(a, A)}=1,969, \mathrm{p}=.168$} & \multirow[t]{2}{*}{ NS } \\
\hline & & Contrôle & 0,008 & 0,01 & & \\
\hline \multirow{2}{*}{$\begin{array}{l}\text { TYPE DE } \\
\text { TEXTE }\end{array}$} & \multirow[t]{2}{*}{43} & Expo. & 0,006 & 0,01 & \multirow[t]{2}{*}{$\mathrm{F}_{(a, A)}=0,062, \mathrm{p}=.804$} & \multirow[t]{2}{*}{ NS } \\
\hline & & Narr. & 0,005 & 0,01 & & \\
\hline
\end{tabular}

Les résultats montrent qu'il n'y a pas de différence significative entre les deux groupes et les types de textes.

\subsubsection{Nombre de révisions selon leur portée}

Des tests de comparaison de moyennes (ANOVA) ont été effectués sur les proportions de révisions (selon le nombre de mots par texte), selon leur nature et selon les facteurs groupe et type de texte (Tableau 8).

Tableau 8. Proportion de révisions par texte selon leur nature et les facteurs GROUPE et TYPE DE TEXTE

\begin{tabular}{|c|c|c|c|c|c|c|}
\hline Facteurs & $\mathrm{N}=$ & & Moyennes & Écart-type & ANOVA & \\
\hline \multicolumn{7}{|c|}{ Révision de nature « ponctuation » } \\
\hline \multirow[t]{2}{*}{ GROUPE } & \multirow[t]{2}{*}{43} & Dys. & 0,008 & 0,01 & \multirow[t]{2}{*}{$\mathrm{F}_{(a, A)}=0,355, \mathrm{p}=.555$} & \multirow[t]{2}{*}{ NS } \\
\hline & & Contrôle & 0,009 & 0,01 & & \\
\hline \multirow{2}{*}{$\begin{array}{l}\text { TYPE DE } \\
\text { TEXTE }\end{array}$} & \multirow[t]{2}{*}{43} & Expo. & 0,009 & 0,01 & \multirow[t]{2}{*}{$F_{(a, 4)}=0,250, p=.620$} & \multirow[t]{2}{*}{ NS } \\
\hline & & Narr. & 0,008 & 0,01 & & \\
\hline \multicolumn{7}{|c|}{ Révision de nature « orthographique » } \\
\hline \multirow[t]{2}{*}{ GROUPE } & \multirow[t]{2}{*}{43} & Dys. & 0,03 & 0,007 & \multirow{2}{*}{$\mathrm{F}_{(a, A)}=9,457, \mathrm{p}=.004$} & \multirow[t]{2}{*}{$\mathbf{S}$} \\
\hline & & Contrôle & 0,015 & 0,007 & & \\
\hline \multirow{2}{*}{$\begin{array}{l}\text { TYPE DE } \\
\text { TEXTE }\end{array}$} & \multirow[t]{2}{*}{43} & Expo. & 0,02 & 0,01 & \multirow[t]{2}{*}{$\mathrm{F}_{(1, t \mid 1)}=2,212, \mathrm{p}=.145$} & \multirow[t]{2}{*}{ NS } \\
\hline & & Narr. & 0,02 & 0,01 & & \\
\hline \multicolumn{7}{|c|}{ Révision concernant le mot } \\
\hline \multirow[t]{2}{*}{ GROUPE } & \multirow[t]{2}{*}{43} & Dys. & 0,01 & 0,007 & \multirow{2}{*}{$\mathrm{F}_{(\mathrm{at1})}=1,063, \mathrm{p}=.309$} & \multirow[t]{2}{*}{ NS } \\
\hline & & Contrôle & 0,009 & 0,007 & & \\
\hline \multirow{2}{*}{$\begin{array}{l}\text { TYPE DE } \\
\text { TEXTE }\end{array}$} & \multirow[t]{2}{*}{43} & Expo. & 0,01 & 0,01 & \multirow[t]{2}{*}{$\mathrm{F}_{(a, A)}=0,801, \mathrm{p}=.019$} & \multirow[t]{2}{*}{ NS } \\
\hline & & Narr. & 0,01 & 0,005 & & \\
\hline \multicolumn{7}{|c|}{ Révision concernant supérieur au mot } \\
\hline \multirow[t]{2}{*}{ GROUPE } & \multirow[t]{2}{*}{43} & Dys. & 0,003 & 0,005 & \multirow[t]{2}{*}{$\mathrm{F}_{(a, 4)}=0,497, \mathrm{p}=.485$} & \multirow[t]{2}{*}{ NS } \\
\hline & & Contrôle & 0,004 & 0,002 & & \\
\hline \multirow{2}{*}{$\begin{array}{l}\text { TYPE DE } \\
\text { TEXTE }\end{array}$} & \multirow[t]{2}{*}{43} & Expo. & 0,005 & 0,007 & \multirow[t]{2}{*}{$\mathrm{F}_{(a, A)}=4,249, \mathrm{p}=.046$} & NS \\
\hline & & Narr. & 0,0019 & 0,004 & & \\
\hline
\end{tabular}


Les résultats montrent que seul le facteur GROUPE a un effet significatif sur la proportion totale des révisions de type orthographique, à savoir des révisions ayant pour objet des corrections orthographiques lexicales et/ou grammaticales (3). Les étudiants dyslexiques en réalisent davantage que les étudiants contrôles.

(3) Puis finissant par la violence ply -> physique [...] » (étudiant contrôle $n^{\circ} 76$, texte narratif)

\subsection{Discussion intermédiaire}

Les analyses quantitatives présentées ci-dessus concernant les révisions textuelles montrent qu'il existe peu de différences significatives entre les étudiants dyslexiques et les étudiants contrôles. En effet, la différence de proportion globale de révisions dans les textes entre les étudiants dyslexiques et contrôles n'est pas significative : les étudiants dyslexiques révisent autant que leurs pairs, ce qui va à l'encontre de notre hypothèse H1. Horowitz-Kraus et Breznitz (2011) ont suggéré que les personnes souffrant de dyslexie ont un déficit dans le mécanisme de détection d'erreur. Ainsi, nos résultats peuvent sembler surprenants. Néanmoins si les étudiants dyslexiques révisent autant leur texte que les étudiants contrôles, il n'en reste pas moins qu'ils font davantage d'erreurs orthographiques et grammaticales que les étudiants contrôles (Mazur-Palandre, 2018, 2019). Ils ont donc des textes finis contenant plus d'erreurs que les étudiants contrôles: les étudiants dyslexiques devraient alors proportionnellement réussir à réviser plus de mots que leurs pairs pour parvenir à un produit final de qualité comparable en termes d'orthographe.

Nos analyses montrent également que les étudiants dyslexiques réalisent le même pattern de révisions que les étudiants contrôles, ce qui confirme notre hypothèse $\mathrm{H} 2$ et va dans le sens d'études antérieures (Farmer et al., 2002). En effet, concernant la distance des révisions, les deux groupes font préférentiellement des révisions courtes, puis moyennes et plus marginalement longues. Concernant le type de révisions, les étudiants réalisent préférentiellement des suppressions, des reformulations et plus rarement des insertions. Concernant la nature des révisions, les analyses montrent que les deux groupes d'étudiants portent leur révision d'abord sur l'orthographe lexicale et grammaticale, puis sur le mot, la ponctuation et marginalement sur des aspects concernant des unités supérieures aux mots.

Les seules différences concernant les deux groupes relèvent du type (suppression, reformulation ou insertion d'une unité) et de la portée (est-ce une révision concernant la ponctuation, l'orthographe, un mot ou plus d'un mot ?) de révisions réalisées. Les étudiants dyslexiques réalisent significativement plus de révisions consistant à remplacer une unité par une autre ( «mais devant l'incivilité absolue de <mes> ces jeunes », étudiant $n^{\circ} 46$ ) et plus de révisions concernant l'orthographe que les étudiants contrôles. Les étudiants dyslexiques, malgré une automatisation moindre du code orthographique, semble réviser ainsi autant que les étudiants contrôles, tout en réalisant davantage de révisions de type reformulation et de nature orthographique. Néanmoins, leurs processus de révisions n'est pas aussi efficace que cela peut paraitre, : ils produisent en effet dans leurs productions écrites, beaucoup plus d'erreurs orthographiques et grammaticales que les étudiants contrôles (Mazur-Palandre, 2018 , 2019) et devraient alors en corriger davantage.

Afin d'apporter un meilleur éclairage sur les processus de révisions de nos participants, et leur niveau d'efficacité chez les personnes dyslexiques, nous proposons ci-après des analyses plus fines sur les résultats des révisions.

\subsection{Efficacité des révisions : forme correcte ou incorrecte ?}

Afin de nuancer ces premiers résultats quantitatifs, des analyses plus fines ont été réalisées. C'est ainsi que nous avons observé toutes les révisions orthographiques et les reformulations, 
afin d'évaluer leur résultat (Tableau 10) : est-ce que ces révisions aboutissent à une forme correcte ou non?

Tableau 10. Pourcentage des révisions résultant à une forme correcte ou incorrecte

\begin{tabular}{lcc}
\hline & $\begin{array}{l}\text { Étudiants } \\
\text { contrôles }\end{array}$ & $\begin{array}{l}\text { Étudiants } \\
\text { dyslexiques }\end{array}$ \\
\hline Révision d'une forme incorrecte à une forme correcte & $58,9 \%(63)$ & $60,5 \%(109)$ \\
\hline Révision d'une forme incorrecte à une forme incorrecte & $1,8 \%(2)$ & $14,5 \%(26)$ \\
\hline Révision d'une forme correcte en une forme correcte & $39,3 \%(42)$ & $25 \%(45)$ \\
\hline Total & $100 \%(107)$ & $100 \%(180)$ \\
\hline
\end{tabular}

Ces analyses révèlent que les révisions orthographiques et de reformulation n'aboutissent pas toujours à une correction. En effet, 14,5\% des révisions des étudiants dyslexiques génèrent des formes erronées, contre seulement $1,8 \%$ chez les étudiants contrôles. Ces résultats vont dans le sens de travaux antérieurs (Morken et Helland, 2013) qui montrent que, si les enfants de 11 ans dyslexiques révisent autant leur texte et de la même manière que les enfants contrôles du même âge, il résulte de ces révisions un produit final de qualité moindre. De plus, nos analyses révèlent que les étudiants dyslexiques se concentrent sur certaines corrections plus que d'autres, ce qui les différencie également des étudiants contrôles. Alors que les étudiants contrôles ont une grande variété de révisions orthographiques, beaucoup des révisions des étudiants dyslexiques concernent : les homophones (a/à ; son/sont ; etc.), les lettres visuellement proches $(b / p ; p / q)$ et la confusion des formes participé passé et infinitive des verbes du premier groupe (mangé / manger). Ces types de révisions semblent efficaces dans la mesure où une étude antérieure révèle que ces mêmes étudiants dyslexiques font peu d'erreurs concernant les homophones et les lettres proches graphiquement (MazurPalandre, 2018, 2019). Ce résultat suggère également que ce type de révision pourrait être une stratégie mise en place durant leur prise en charge.

\section{Conclusion}

Les étudiants dyslexiques réussissent donc à aller au-delà de leurs difficultés à l'écrit pour réviser leur texte en détectant et corrigeant certaines de leurs erreurs. Néanmoins, nos résultats suggèrent que les étudiants dyslexiques révisent leur texte de manière moins efficace que leurs pairs, en détectant plus des erreurs qui peuvent ne pas en être et en faisant plus d'erreurs de correction, que les étudiants contrôles. De plus, proportionnellement à leur nombre d'erreurs les étudiants dyslexiques devraient réaliser davantage de révisions que les étudiants contrôles qui font beaucoup moins d'erreurs dans leur texte. Réviser les productions écrites n'empêche pas les erreurs d'orthographe chez les étudiants dyslexiques. Ces résultats confirment également les ressentis des étudiants dyslexiques qui disent ressentir plus de difficultés en production textuelle que les étudiants contrôles (Tableau 4). Les analyses statistiques du questionnaire révèlent également que les étudiants dyslexiques interrompent régulièrement leur activité d'écriture à cause de leur fatigabilité et des bruits environnants, ce qui peut avoir un impact sur la révision textuelle. Les étudiants dyslexiques semblent alors bien se connaitre en tant que lecteur/scripteur en identifiant leurs difficultés, ce qui est une réelle force. Des études ont en effet démontré qu'une meilleure connaissance leur dyslexie (Carter et Sellman, 2013 ; Pino et Mortari, 2014 ; Raskind et al., 1999), une conscientisation du trouble ainsi que l'adoption et l'éducation à des pratiques sociales de l'écriture (Carter et Sellman, 2013), la prise en main et l'acceptation de son trouble rend les activités d'écriture moins pénibles et améliorent l'apprentissage (Pino et Mortari, 2014). Il est ainsi important, en plus d'analyses de bilans orthophonique et neuropsychologique, d'expérimentations psycholinguistiques et de questionnaires, de prendre en considération et s'intéresser vivement à la trajectoire de l'individu dyslexique (Mazur-Palandre et Witko, 2019a, 2019b), qui est 
évolutive, afin de mieux percevoir ces facteurs relevant plus de l'intériorisation du trouble que des déficits en eux-mêmes (Witko, 2019).

Nous tenons à remercier : Raphaëlle Abadie grâce à qui la collecte des données s'est parfaitement déroulée et pour son expertise en tant que neuropsychologue ; Nathalie Bedoin pour son expertise en psychologie cognitive ; Florence Chenu pour, entre autres, son aide technique (création de filtres pour l'export dans CLAN) ; Louis Maritaud pour son minutieux codage des données ; Céline Faure (MSH) pour ses conseils statistiques ; les étudiants volontaires ; le Laboratoire d'Excellence ASLAN, le CNRS, ICAR (UMR 5191) et l'ENS de Lyon pour leur soutien financier.

\section{Références bibliographiques}

Abadie, R. et Bedoin, N. (2016). Les étudiants dyslexiques à l'université. Quels déficits cognitifs et langagiers? Neurologies, 192, 298-303.

American Psychiatric Association. (2013). Diagnostic and statistical manual of mental disorders DSM5 (5e éd.). Arlington, VA : American Psychiatric Publishing.

Beaton, A., McDougall, S. et Singleton, C. (1997). Dyslexia in literate adult. Journal of research in reading (Special Issue), 20(1).

Bedoin, N. (2014) Dyslexie chez l'enfant et déficits d'attention spatiale. Conférence invitée aux Entretiens d'Orthophonie 2014, Paris.

Bedoin, N. et Médina, F. (2014). Logiciel Sélection et Inhibition des traitements Global et Local, www.gnosia.fr.

Bereiter, C. et Scardamalia, M. (1987). The psychology of written composition. Hillsdale: Lawrence Erlbaum.

Berman, R. (1998). Typological perspectives on connectivity, In Penner, Z., Dittmar, N. (Eds.), Issues in the theory of language acquisition, 203-224. Bern: Peter Lang.

Berman, R. A. et Nir-Sagiv, B. (2009). Clause-packaging in narratives: A crosslinguistic developmental study. In J. Guo, E. Lieven, S. Ervin-Tripp, N. Budwig, S. Özçalişkan, K. Nakamura (eds.), Crosslinguistic approaches to the psychology of language: Research in the tradition of Dan I. Slobin, pp. 149-162. NY: Taylor \& Francis.

Berninger, V. et Swanson, H. (1994). Modifying Hayes and Flower's model of skilled writing to explain beginning and developing writing. In Carlson, J., Buttterfly, E. (Eds.), Advances in Cognition and Educational Practice, Children's Writing: Toward a Process Theory of the Development of Skilled Writing, Vol. 2, 57-8. Greenwich: J.A.I. Press.

Bosse, M. L., Tainturier, M. J. et Valdois, S. (2007). Developmental dyslexia: The visual attention span deficit hypothesis. Cognition, 104(2), 198-230.

Bosse, M. L. et Valdois, S. (2009). Influence of the visual attention span on child reading performance: A cross-sectional study. Journal of Research in Reading, 32, 230-253.

Boutard, C., Claire, I., et Gretchanovsky. L. (2004) Le vol du PC : évaluation fonctionnelle de la lecture chez les sujets de 11 à 18 ans. Isbergues : Ortho Editions.

Carter, C. et Sellman, E. (2013). A View of Dyslexia in Context: Implications for Understanding Differences in Essay Writing Experience Amongst Higher Education Students Identified as Dyslexic. Dyslexia 19(3), 149-164.

Chenu F., Jisa H. et Mazur-Palandre A. (2012). Développement de la connectivité syntaxique à travers deux types de textes à l'oral et à l'écrit. In F. Neveu, V. Muni Toke, P. Blumenthal, T. Klingler, P. Ligas, S. Prévost, S. Teston-Bonnard (Eds.), Actes de la Xème Congrès Mondial de la Linguistique Française, pp. 1591-1605. Institut de Linguistique Française.

Chesnet, D. et Alamargot, D. (2005). Analyses en temps réel des activités oculaires et graphomotrices du scripteur: intérêt du dispositif 'Eye and Pen'. L'Année Psychologique, 105, 477-520.

Farmer, M., Riddick, B., \& Sterling, C. (2002). Dyslexia and inclusion: assessment and support in higher education. London and Philadelphia: Whurr Publishers.

Fayol, M. et Miret, A. (2005). Écrire, orthographier et rédiger des textes, Psychologie Française, 50, 391-402.

Gola-Asmussen, C., Lequette, C., Pouget, G., Rouyer, C. et Zorman, M. (2010). Outil d'évaluation de compétences de lecture chez l'adulte de plus de 16 ans. Université de Provence Aix-Marseille 1 et Cognisciences LES Université Pierre Mendès.

Giménez, A., Luque, J. L., Lopez-Zamora, M. et Fernandez-Navas, M. (2015). A self-report of reading disabilities for adults: ATLAS. Anales de Psicologia, 31(1), 109-119. 
Gregg, N., Coleman, C., \& Lindstrom, L. (2008), Assessment of written expression in the adult population. In L. Wolf, H. Schreiber, \& J. Wasserstein (Eds.), Adult learning disorders: contemporary issues (pp. 301-331). New York Hove: Psychology Press.

Hacker, D.J. Plumb, C. Butterfield, E.C., Quathamer D. et Heineken, E. (1994). Text revision : detection and correction of errors. Journal of Educational Psychology, 86, 65-78.

Hatcher, J., Snowling, M., \& Griffiths, Y. (2002). Cognitive assessment of dyslexic students in higher education. British Journal of Educational Psychology, 72, 119-133.

Habib M. (2003). La dyslexie à livre ouvert. Résodys, Marseille.

Habib, M. (2018). Dyslexie de développement, EMC, Psychiatrie/Pédopsychiatrie, 0(0), 1-12.

Hayes, J. (1995). Un nouveau modèle du processus d'écriture, In Boyer, J.Y., Dionne, J.P. (Eds.), La production de textes : vers un modèle d'enseignement de l'écriture, 49-72. Montréal: Logiques.

Hayes, J. (1998). Un nouveau cadre pour intégrer cognition et affect dans la rédaction, In Piolat, A., Pélissier, A. (Eds.), la rédaction de textes, 51-101. Lausanne: Delachaux et Niestlé.

Hayes, J. R., \& Berninger, V. W. (2014). Cognitive processes in writing: A framework. Writing development in children with hearing loss, dyslexia, or oral language problems, 3-15.

Hayes J. R. \& Flower L. (1980). « Identifying the organization of writing processes », In L.W. Gregg \& E.R. Sternberg (éd.) Cognitive processes in writing. Hillsdale, NJ : Laurence Erlbaum Associates Inc, 3-30.

Horowitz-Kraus, T. et Breznitz, Z. (2011). Error detection mechanism for words and sentences: a comparison between readers with dyslexia and skilled orders. International Journal of Disability, Development and Education, 58(1), 33-45.

Hunt, K. (1970). Syntactic Maturity in School children and Adults, Monographs of the Society for Research in Child Development, 35, iii-67.

Jisa, H. (2004). Growing into academic French. In Berman, R. (Eds.), Later Language Development: Typological and Psycholinguistic Perspectives, (TiLAR), 135-161. Amsterdam : John Benjamins.

Jisa, H., Ailhaud, E., Chenu, F. et Mazur-Palandre, A. (2016). L'influence de la langue écrite au cours du développement langagier tardif. Actes éditées du CMLF 2016.

Just, M. A., \& Carpenter, P. A. (1992). A capacity theory of comprehension: Individual differences in working memory. Psychological Review, 99(1), 122-149.

Kellogg, R. (1994). The psychology of writing. New York: Oxford University Press.

Lyon, G. R., Shaywitz, S. E., \& Shaywitz, B. A. (2003). Defining dyslexia, comorbidity, Teachers' knowledge of language and reading. A definition of dyslexia. Annals of Dyslexia, 53, 1-14.

McCutchen, D. (1996). A capacity theory of writing: Working memory in composition, Educational Psychology Review, 8, 299-325.

Mazur-Palandre, A. et Jisa, H. (2013). La complexité lexicale des syntagmes nominaux : Une étude développementale. Enfance, 4, 359-371.

Mazur-Palandre, A. (2018). La dyslexie à l'âge adulte : la persistance des difficultés orthographiques. In F. Neveu, B. Harmegnies, L. Hriba et S. Prévost (Eds.), Actes édités du 6ème Congrès Mondial de Linguistique Française, 46, 10003. Institut de Linguistique Française ; EDP Sciences.

Mazur-Palandre, A. (2019). Les étudiants diagnostiqués dyslexiques durant leur enfance présentent-ils encore des difficultés dans l'enseignement supérieur? L'Orthophoniste, 391, 14-18.

Mazur-Palandre, A. et A. Witko (2019a). La dyslexie à l'âge adulte : un trouble persistant et déroutant. L'Orthophoniste, 391, 13-14.

Mazur-Palandre, A. et A. Witko (2019b). La dyslexie : vers une définition transdisciplinaire ?. L'Orthophoniste, 391, 24-24.

Mazur-Palandre, A., Abadie, R. et Bedoin, N. (2016). Étudiants dyslexiques à l'Université : Spécificités des difficulté ressenties et évaluation des déficits. In M. Habib (Ed.), 141-179. De Boeck.

Morken, F. et Helland, T. (2013). Writing in Dyslexia: Product and Process. Dyslexia, 19:131-148.

Mosenthal, P. (1985). Defining the expository discourse continuum toward a taxinomy of expository text, Poetics, 14, 387-414.

Pino, M. et Mortari, L. (2014). The inclusion of students with dyslexia in higher education: a systematic review using narrative synthesis.Dyslexia, 20(4), 246-69.

Raskind, M.H. et Higgins, E. (1995). Effects of speech synthesis on the proofreading efficiency of postsecondary students with learning disabilities. Learning Disability Quarterly, 18, 141-58.

Riddick, B., Farmer, M. et Sterling, C. (1997) Students and dyslexia: Growing up with specific learning difficulty. London: Whurr. 
Rüsseler, J., Becker, P., Johannes, S. et Münte, T.F. (2007). Semantic, syntactic, and phonological processing of written words in adult developmental dyslexic readers: an event-related brain potentialstudy. BMC Neuroscience, 8(52).

Seidenberg, M.S. et McClelland, J.L. (1989). A distibuted, developmental model of word recognition and naming. Psychological Review, 96(4), 523-568.

Singleton, C.H. (2001). Dyslexie in Higher Education : Policy, Provision and Practice. Report of the National Working Party on dyslexia in higher Education. Hull : University of Hull.

Snowling, M. (2000). Dyslexia (2nd ed.). Oxford: Blackwell.

Sterling, C., Farmer, M. Riddick, B., Morgan, S. et Matthews, C. (1998). Adult dyslexic writing. Dyslexia, 4, 1-15.

Stein, J. (2001). The magnocellular theory of developmental dyslexia. Dyslexia, 7(1), 12-36.

Torrance, M. et Galbraith, D. (2006). The processing demands of writing, In MacArthur, C., Graham, S., Fitzgerald, J. (eds.), Handbook of Writing Research, 67-82, New York: The Guilford Press.Valdois, S., Guinet, E., et Embs, J.-L. (Producer). EVADYS. Isbergues : Ortho Editions.

Witko A. (2019). La dyslexie à l'âge adulte : comment révéler son potential pour dépasser son trouble ? L'Orthophoniste, 391, 18-20.

Zimmermann, P. et Fimm, B. (2012). Testbatterie zur Aufmerksamkeitsprüfung - Version Mobilität (Test battery for the assessment of attentional skills-Mobility version). Herzogenrath: Psytest. 